VOL. $15(1976), 461-465$.

\title{
The category of uniform convergence spaces is cartesian closed
}

\author{
R.S. Lee
}

\begin{abstract}
This paper first assigns specific uniform convergence structures to the products and function spaces of pairs of uniform convergence spaces, and then establishes a bijection between corresponding sets of morphisms which yields cartesian closedness.
\end{abstract}

\section{Introduction}

Cartesian closed categories, being set-like, are useful in topology. Extensive bibliographies exist in Herrlich [3], Nel [5], and Wyler [6]. For a category that is not cartesian closed, embedding into one may be considered. For example: the category of uniform spaces is known to be not cartesian closed, but it can be embedded into the category $U$ of uniform convergence spaces. This paper shows that $U$ is cartesian closed other properties of $U$ will be discussed in a future article.

The definition of uniform convergence structure, given below and used throughout this paper, is due to Wyler [7], since the uniform convergence structure introduced by Cook and Fischer [1] contains the diagonal filter; thus, cannot be used if a function space with the continuous convergence structure is to be in $U$, as shown by Example 2.2 in Gazik, Kent, and Richardson [2].

\section{Uniform convergence spaces}

NOTATION. $[B]$ denotes the filter generated by the filter base $B$.

Received 4 August 1976. The author wishes to thank Professor Oswald Wyler for suggesting this problem and for kindly giving directions and advice. 
Let $F$ and $G$ be two filters on $X \times X$ including null filters [7]. Then $F^{-1}=\left[\left\{F^{-1} \mid F \in F\right\}\right]$ where $F^{-1}=\{(a, b) \mid(b, a) \in F\} ;$ and $F \circ G=[\{F \circ G \mid F \in F, G \in G\}]$ where

$$
F \circ G=\{(a, c) \mid \text { there exist } b \in X \text { with }(a, b) \in F \text { and }(b, c) \in G\}
$$

represents the usual composition of relations in the reverse order. $\dot{x}$ denotes the ultrafilter generated by $\{x\}$. For every filter $F$ of $U(Y, Z) \times U(Y, Z)$, and $G$ of $Y \times Y$, together with the evaluation map $e: U(Y, Z) \times Y \rightarrow Z$, we define $(e \times e)(F \times G)$ to be the filter $F(G)=[\{F(G) \mid F \in F, G \in G\}]$ where

$$
F(G)=\{(f a, g b) \mid(f, g) \in F,(a, b) \in G\} .
$$

Using the order notation of Kowalsky and Wyler, a filter $F$ is less than or equal to a filter $G$ if $F$ is finer than $G$. Also, $F \cup G$ denotes the filter $\{F \cup G \mid F \in F, G \in G\}$, which is the set-intersection of $F$ and $G$; and $F \cap G$ denotes the filter $\{F \cap G \mid F \in F, G \in G\}$ which is generated by the set-union of $F$ and $G$. These symbols of filter operations carry over formal laws from set algebra to filter algebra in a natural way; see Wyler [7].

A uniform convergence structure $J$ on a set $X$ is a set of filters on $X \times X$ such that

$$
\begin{aligned}
& \text { (i) } \dot{x} \times \dot{x} \in J \text { for every } x \in X \text {; } \\
& \text { (ii) if } F \in J \text { and } G \leq F \text {, then } G \in J \text {; } \\
& \text { (iii) if } F, G \in J \text {, then } F \cup G \in J \text {; } \\
& \text { (iv) if } F \in J \text {, then } F^{-1} \in J \text {; } \\
& \text { (v) if } F, G \in J \text {, then } F \circ G \in J .
\end{aligned}
$$

A uniform convergence space is a pair $(X, J)$ of a set $X$ and a uniform convergence structure $J$ on $X$. A map $f$ from a uniform convergence space $(X, J)$ to a uniform convergence space $(Y, K)$ is uniformly continuous iff $(f \times f) \mathrm{J} \subset K$.

The collection $U$ of all uniform convergence spaces and uniformly continuous maps form a category, the identity map for each uniform convergence space and the composition of uniformly continuous maps being uniformly continuous [1]. 
Let $V$ be the initial structure on $X \times Y$ with respect to uniformly continuous projections $p$ and $q$ from the product to $X$ and $Y$, respectively. Then $V$ consists of all filters on $(X \times Y) \times(X \times Y)$ generated by $(p \times p)^{-1} F \cap(q \times q)^{-1} G$ for filters $F \in J, G \in K$, and is known as the product uniform convergence structure [1].

LEMMA 1. $(X \times Y, V)$ is a product of $(X, J)$ and $(Y, K)$ in the category $U$.

Proof. By Theorems 12 and 14 in [1].

Let $U(X, Y)$ be the set of all uniformly continuous maps from $(X, J)$ to $(Y, K)$, and $W$ be the uniform continuous convergence structure on $U(X, Y)$; specifically, $W$ is the set of all filters $H$ on $U(X, Y) \times U(X, Y)$ such that $(e \times e)(H \times F) \in K$ for every $F \in J$.

LEMMA 2. W is a zoniform convergence structure of $U(X, Y)$.

Proof. $(e \times e)((\dot{f} \times \dot{f}) \times F)=(f \times f)(F) \in K$ for every $f \in U(X, Y)$ and $F \in J, f$ being uniformly continuous. Thus $\dot{f} \times \dot{f} \in W$. Suppose $A \in W$, and $B \leq A$; then for every $F \in J, B(F) \leq A(F)$ in $K$. Hence $B \in W$. If $A, B$ in $W$, then $(A \cup B)(F)=A(F) \cup B(F)$ in $K$ for every $F \in J$, showing that $A \cup B \in W$. For every $A \in W$,

$$
A^{-1}(F)=\left(A\left(F^{-1}\right)\right)^{-1}
$$

$F^{-1} \in J$, therefore $A\left(F^{-1}\right) \in K$ which implies that $\left(A\left(F^{-1}\right)\right)^{-1} \in K$. For $A, B$ in $\omega,(A \circ B)(F) \leq A(F) \circ B\left(F^{-1} \circ F\right) \in K$, since $(A \circ B)(F) \subseteq A(F) \circ B\left(F^{-1} \circ F\right)$, where $A \in A, B \in B$, and $F \in F$. Hence $A \circ B \in W$.

LEMMA 3. For each $(Y, K)$ in $U$, the functor $-\times Y: X \mapsto X \times Y$ is a left adjoint to $U(Y,-): Z \mapsto U(Y, Z)$, whenever $X \times Y$ and $U(Y, Z)$ have the respective structures $v$ and $w$ used in Lemmas 1 and 2.

Proof. By Lemma $1,-\times Y$ is an endofunctor of $U$. The adjoint situation is shown below by an equivalent condition [4], which states that for each uniform convergence space $(Z, L)$ the corresponding evaluation map $e: U(Y, Z) \times Y \rightarrow Z$ is a universal arrow from the functor $-\times Y$ to $Z$. For every uniform convergence space $(X, J)$ and $f: X \times Y \rightarrow Z$, define $f^{*}: X \rightarrow U(Y, Z)$ by $\left(f^{*}(x)\right)(y)=f(x, y)$. At the set-level, 
$f=e\left(f^{*} \times\right.$ Id $\left.Y\right)$, where Id $Y$ is the identity map of $Y$. For every filter $F \in J$, and $G \in K$,

$\left(\left(f^{*} \times f^{*}\right)(F)\right)(G)=(e \times e)\left(\left(f^{*} \times f^{*}\right)(F) \times G\right)$ $=(e \times e)\left(\left(\left(f^{*} \times \operatorname{Id} Y\right) \times\left(f^{*} \times \operatorname{Id} Y\right)\right)(H)\right)=(f \times f)(H)$,

where $H=(p \times p)^{-1} F \cap(q \times q)^{-1} G$ is a filter in $V$. Since we give $U(Y, Z)$ the uniform continuous convergence structure, $e$ is uniformly continuous. Hence, if $f$ is uniformly continuous, we have $\left(f^{*} \times f^{*}\right)(F) \in W$ for every $F \in J$, which shows that $f^{*}$ is uniformly continuous. On the other hand, if $f^{*}$ is uniformly continuous, clearly so is $f$.

THEOREM. The category $U$ of uniform convergence spaces and uniformly continuous maps is cartesian closed.

Proof. $U$ has a terminal object, namely a singleton uniform convergence space, has products by Lemma 1 , and satisfies Lemma 3 . Hence $U$ is cartesian closed.

\section{References}

[1] C.H. Cook and R.H. Fischer, "Uniform convergence structures", Math. Ann. 173 (1967), 209-306.

[2] R.J. Gazik, D.C. Kent, and G.D. Richardson, "Regular completions of uniform convergence spaces", Bull. Austral. Math. Soc. 11 (1974), 413-424.

[3] Horst Herrlich, "Cartesian closed topological categories", Math. Colloq. Univ. Cope Toun 9 (1974), 1-16.

[4] Saunders Mac Lane, Categories for the working mathematicicon (Graduate Text in Mathematics, 5. Springer-Verlag, New York, Heidelberg, Berlin, 1971).

[5] L.D. Nel, "Cartesian closed topological categories", Categorical topology, 439-451 (Proc. Conf. Mannheim, 1975. Lecture Notes in Mathematics, 540. Springer-Verlag, Berlin, Heidelberg, New York, 1976). 
[6] Oswald Wyler, "Convenient categories for topology", General Topology and App I. 3 (1973), 225-242.

[7] Oswald Wyler, "Filter space monads, regularity, completions", TOPO 1972 - General topology and its applications, 591-637 (Second Pittsburgh International Conference, 1972. Lecture Notes in Mathematics, 378. Springer-Verlag, Berlin, Heidelberg, New York, 1974).

Department of Mathematics,

Duquesne University,

Pittsburgh,

Pennsyl vania,

USA. 